\title{
Novel ARID1B variant inherited from somatogonadal mosaic mother in siblings with Coffin-Siris syndrome 1
}

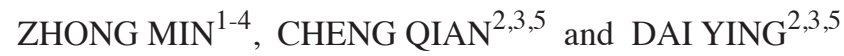 \\ ${ }^{1}$ Department of Neurology, Children's Hospital of Chongqing Medical University; ${ }^{2}$ Pediatric Research Institute, \\ China International Science and Technology Cooperation Base of Child Development and Critical Disorders, \\ Chongqing Engineering Research Center of Stem Cell Therapy, Ministry of Education Key Laboratory of \\ Child Development and Disorders; ${ }^{3}$ National Clinical Research Center for Child Health and Disorders, \\ Chongqing 400014; ${ }^{4}$ Department of Pediatric, Qianjiang Central Hospital of Chongqing, Chongqing 409000; \\ ${ }^{5}$ Department of Primary Child Health Care, Children's Hospital of Chongqing Medical University, \\ Chongqing 400014, P.R. China
}

Received September 10, 2019; Accepted April 21, 2020

DOI: $10.3892 /$ etm.2021.10046

\begin{abstract}
Coffin-Siris syndrome1 (CSS1; Online Mendelian Inheritance in Man no. 135900) is a multiple malformation syndrome characterized by intellectual and/or developmental delay, and hypoplastic or absent fifth fingernails and/or toenails. AT-rich interaction domain-containing protein 1B (ARID1B) is the most frequently mutated gene in CSS1 and the majority of reported cases have been sporadic. Using whole-exome sequencing, the present study identified two siblings with CSS1 with a novel heterozygous co-segregating pathogenic variant in the ARID1B gene (c.3468_3471del). Additionally, the current study confirmed a 4\% somatic ARID1B mosaicism in the patient's mother. The results expanded the spectrum of known ARID1B pathogenic variants. To the best of our knowledge, the present study is the first to provide experimental evidence that an ARID1B pathogenic variant can be inherited from a clinically healthy somatogonadal mosaic mother.
\end{abstract}

\section{Introduction}

Coffin-Siris syndrome1 [CSS1; Online Mendelian Inheritance in Man (OMIM) no. 135900] was first described as a multiple malformation syndrome in 1970 and is characterized by intellectual and/or developmental delay and hypoplastic or absent fifth fingernails and/or toenails (1-3). Haploinsufficiency of the AT-rich interaction domain-containing protein 1B (ARID1B) has been reported to cause CSS1 (4). The ARID1B variant is

Correspondence to: Dr Dai Ying, Department of Primary Child Health Care, Children's Hospital of Chongqing Medical University, 136 Zhongshan 2nd Road, Chongqing 400014, P.R. China

E-mail: dai@hospital.cqmu.edu.cn

Key words: AT-rich interaction domain-containing protein 1B, variant, Coffin-Siris syndrome, whole-exome sequencing, mosaicism reported to be the most frequently mutated gene in CSS (3,5-7) and the majority of reported cases are sporadic pathogenic variants $(5,8)$.

ARID1B encodes a component of the Brahma-associated factor complex, also known as the mammalian SWItch/sucrose non-fermentable (SWI/SNF) complex $(9,10)$. The SWI/SNF complex is an ATP-dependent chromatin remodeling complex, which modifies chromatin structure and facilitates transcription factor access to DNA (9-11). Forms of CSS have been demonstrated to be caused by pathogenic variants in numerous genes encoding subunits of the SWI/SNF complex, including CSS2-8 (OMIM nos. 614607, 614608, 614609, $616938,617808,618027$ and 618362) which are caused by variants in the AT-rich interaction domain-containing protein 1A (ARID1A), SWI/SNF related matrix-associated actin-dependent regulator of chromatic subfamily (SMARC) B member 1 (SMARCB1), SMARCA4, SMARCE1, AT-rich interaction domain-containing protein 2 (ARID2), double plant homeodomain finger 2 (DPF2) and SMARCC2 genes (OMIM nos. 603024, 601607, 603254, 603111, 609539, 601671 and 601734, respectively) $(2,3,5,6)$. A similar phenotype, Nicolaides-Baraitser syndrome (OMIM no. 601358) is caused by a pathogenic variant in a subunit of the SMARCA2 complex (OMIM no. 600014) (3).

Mosaicism is a biological phenomenon that describes an individual who has developed from a single fertilized egg and has two or more populations of cells with distinct genotypes (12). Specific types of mosaicism describe the parts of the body that harbor the variant cells and the potential for transmission to offspring, including germline mosaicism (also known as gonadal mosaicism), somatic mosaicism and somatogonadal mosaicism (a combination of germline and somatic mosaicism) (12-16). Postzygotic mosaicism refers to mutations that result in distinct cell populations within the same individual when somatogonadal mosaicism cannot be fully excluded (14-16). The developmental timing and cell lineage are affected and, combined with the phenotypic consequences of the pathogenic variant, ultimately determine 
the tissue distribution of mosaicism (somatic, germline or somatogonadal), as well as the patterns of disease reoccurrence within families $(12,14)$. Somatogonadal mosaicism that arises at an early embryonic stage can involve both somatic and germ cells, and individuals with somatogonadal mosaicism are at risk of having affected children $(12,14)$.

The present study reported two siblings with CSS1 and demonstrated rare genetic inheritance from their mother with somatogonadal mosaicism.

\section{Materials and methods}

Ethical statement. Ethical approval for the present study was obtained from the Institutional Review Board of the Children's Hospital of Chongqing Medical University, Chongqing, China (ethical approval no. 2018-64). Written informed consent was obtained from the parents of the siblings.

Clinical characterizations. The patient in the present study was a girl aged 6 years and 3 months, born full-term to non-consanguineous Chinese parents. She was delivered normally (gravida 3; para 3) with a birth weight of 4,150 g. The length of the baby and head circumference at birth were unknown. Feeding problems were observed during the infant period. The first baby of the parents had died shortly following birth.

The patient came to hospital and her mother reported that the patient had intellectual and linguistic developmental delays. The patient spoke her first words 'mum mum' at the age of 4 years and had slightly improved; however, she only said 'mum mum' during the clinical evaluation. She began to walk by herself at 2 years old. She has a severe intellectual delay and an IQ $<32$ according to the Wechsler Preschool and Primacy Scale of Intelligence test (17). According to the operation manual, the average score is 100 and the $95 \%$ confidence interval is $70-130$.

Upon examination, the patient weighed $14 \mathrm{~kg}$ (z-score, -2.91), her height was $105.2 \mathrm{~cm}$ (z-score, -2.23), head circumference was $46.5 \mathrm{~cm}$ ( 20-month average) and BMI was 12.7 (z-score, -2.05), all of which are abnormal. She displayed dysmorphic features, including brachytelephalangy and hypoplastic nails of the fifth digit phalanges and fingers, coarse facial features, low hairline, short philtrum, thick lips, mild scoliosis (Fig. 1A-C) and body hypertrichosis. Brain MRI and metabolic screening were normal.

The patient's older brother exhibited similar disease onset and progression. He was 10 years old at the time of the current study and had learning difficulties. Physical examination demonstrated similar dysmorphic features tothe patient, except for moderate scoliosis (Fig. 1B). The parents were clinically healthy and exhibited no dysmorphic features.

Whole-exome sequencing (WES). Peripheral blood samples were collected from the patient and her family in EDTA tubes. The genomic DNA of the patient, her brother and their parents were screened for genetic variations using trio WES. Briefly, the DNA was sheared using an ultrasonic processor (version no. KQ218; Kunshan Ultrasonic Instruments Co., Ltd.) and hybridized with the xGen Exome Research Panel probe sequence capture array (version no. 1.0; Integrated
Device Technology, Inc.) to enrich the exonic region, according to the manufacturer's protocol. Quantitative PCR was performed for effective molecular concentration detection of the exome libraries using a KAPA Library Quantification kit (cat. no. KR0405-v8.17; the kit included SYBR-Green; Kapa Biosystems, Roche Diagnostics), according to the manufacturer's protocol. The primers and thermocycling conditions are presented in Table I. The size distribution and concentration of the exome libraries was tested using Bioanalyzer 2100 (Agilent Technologies, Inc.), according to the manufacturer's protocol. A standard curve was generated and was used to convert the average $\mathrm{Cq}$ score for each library. The average size-adjusted concentration for each library that was assayed was calculated by multiplying the calculated average concentration using the following formula: Size of DNA standard in bp (452)/average fragment length of library in bp.

Sanger sequencing. In order to validate the pathogenic variants detected by WES, the genomic DNA of the patient, her brother and their parents was evaluated using PCR and the products were analyzed using Sanger sequencing. PCR amplification was performed using a KAPA2G Robust HotStart PCR kit (Kapa Biosystems) on a Hema 9600 PCR Thermo Cycler (Zhuhai Hema Medical Instrument Co., Ltd.), according to the manufacturer's protocol. The primers and thermocycling conditions are presented in Table II.

Sequences were assembled and analyzed using the DNASTAR Laser gene software package (version no. 7.1; DNASTAR, Inc.; www.dnastar.com/documentation).

Amplicon-based deep sequencing. A two-stage PCR was performed. For the first stage of PCR, the PCR reaction system was prepared using sample DNA. Each step of the two-stage PCR was identical to that described previously, except for the specific primers (Chigene; not commercially available). The product directly served as the template for the second stage of PCR with the same procedure, except for the second pair specific primers. The primers used in each stage were specifically designed by Chigene (Chigene Translational Medical Research Center Co. Ltd.; chigene.org) and are not commercially available. The purified DNA products underwent Next Generation Sequencing (NGS)-based ultra-deep sequencing (sequencing depth, $>500,000 \mathrm{X}$ ). The sequencing data were then used for bioinformatics analysis.

Bioinformatics analysis. Raw image files were processed using a BCL2FASTQ software package (version no. 1.8.4; Illumina, Inc.; support.illumina.com/content/dam/illumina-support/ documents/documentation/software_documentation/bcl2fastq/ bcl2fastq_letterbooklet_15038058brpmi.pdf) to generate fastq data. Low-quality variations of the quality score $<20$ were filtered out. The sequencing reads were aligned to the National Center for Biotechnology Information (www.ncbi.nlm.nih.gov/) human reference genome using Burrows-Wheeler Aligner (version no. 0.7.11-r1034; https://github.com/lh3/bwa). SAMtools (version no. 0.1.19; github.com/samtools/samtools) and Pindel (version no. 0.2.4; github.com/genome/pindel) software were used to call single-nucleotide variants and indels of the reads. The minor allele frequency (MAF) was annotated using the databases dbSNP (version no. 151; http://www.ncbi.nlm.nih.gov/ 
projects/SNP/), 1,000 Genomes MAF (Chinese; https://www. internationalgenome.org/), Genome Aggregation Database (gnomAD r2.0.2; https://gnomad.broadinstitute.org/), ExAC (merged with the gnomAD database) and an in-house MAF (Chigene; not commercially available) that includes $>100,000$ Chinese exomes. Synonymous substitutions or single-nucleotide variants with MAF $>5 \%$ were filtered out. Transcriptions and translations of non-synonymous variants were predicted using Sorting Intolerant from Tolerant and Protein Variation Effect Analyzer (version no. 1.1.3; http://provean.jcvi.org), Polymorphism Phenotyping (version no. 2; http://genetics.bwh.harvard.edu/pph2/). The pathogenicity of variants was annotated according to the American College of Medical Genetics and Genomics (ACMG) standards and guidelines (18).

\section{Results}

WES. Due to the absence of phenotypes in the parents and the presence of similar phenotypes in the patient and her older brother, the condition was assumed to exhibit an autosomal recessive mode of inheritance. However, evaluation according to ACMG guidelines and clinical features did not present a homozygous or compound heterozygous variant that would have been expected in a recessive inheritance model. Therefore, the condition was hypothesized to exhibit ta dominant inheritance model. The present study therefore identified a de novo heterozygous 4-bp deletion (c.3468_3471del) in the 13th exon of the ARID1B gene (NM_020732; Fig. 2). This deletion is predicted to result in a frame shift and premature termination. To the best of our knowledge, this pathogenic variant in the ARID1B gene has not previously been reported. De novo occurrence was confirmed using Sanger sequencing of samples from the patient and her parents (Fig. 2). No other pathogenic variants were identified in screening of all associated CSS genes (ARID1B, SMARCB1, SMARCA4, SMARCE1, ARID2, DPF2, SMARCC2 and SMARCA2).

Amplicon-based deep sequencing. After excluding the possibility of incomplete penetrance of CSS1, one of the parents was suspected to be a gonadal mosaic. In order to evaluate this hypothesis, amplicon-based deep sequencing was performed using DNA from the peripheral blood of the patient's parents, which confirmed a $4 \%$ somatic ARID1B mosaicism in the patient's mother (Fig. 3). The previous detection of the ARID1B pathogenic variant in the patient's brother indicated the presence of somatogonadal ARID1B mosaicism in the mother.

\section{Discussion}

CSS was first reported by Coffin and Siris (1) and is characterized by notable hypoplasia of the fifth digit phalanges and/or nails, coarse facial features and sparse scalp hair (1). Worldwide, $>100$ CSS cases with ARID1B pathogenic variants have been reported in the last two years and the clinical features have been summarized $(7,8)$. Previous evidence has indicated that the majority of CSS cases exhibit a certain degree of intellectual and/or developmental delay $(4,5,8,19)$. There are two facial features that have been categorized $(4,20)$. Patients with classical CSS have coarse facial features, bushy eyebrows and thick
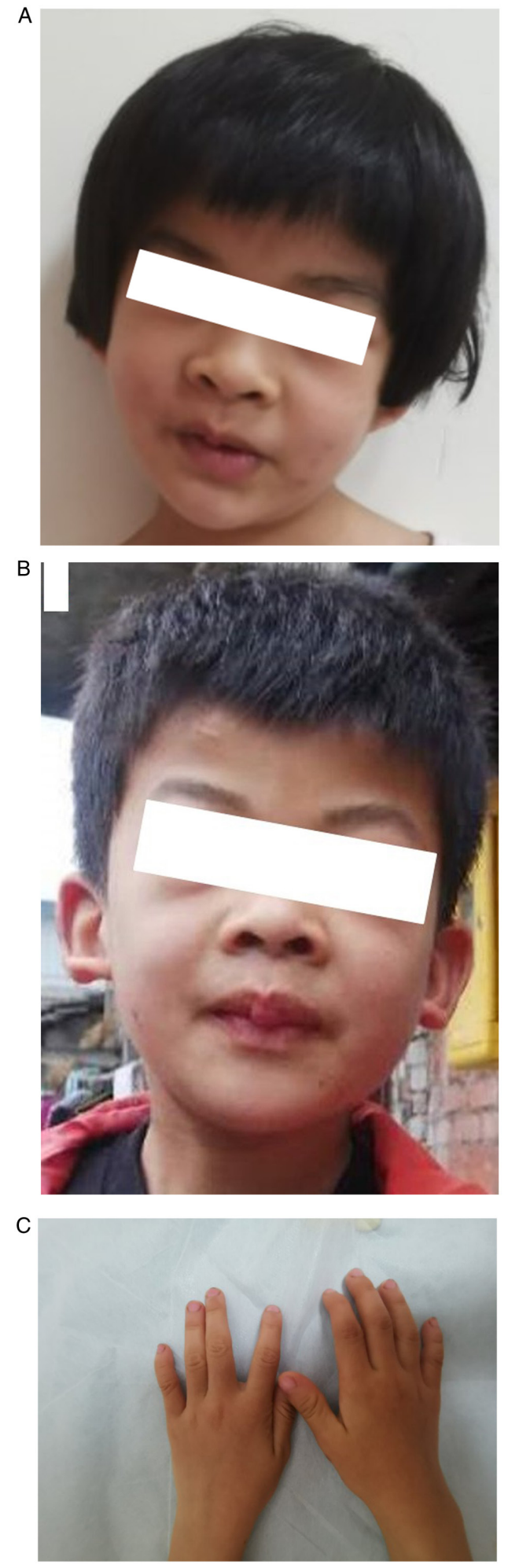

Figure 1. Photograph of (A) the patient and (B) her brother, demonstrating facial dysmorphism, including coarse facial features, low hairline and thick lips. (C) Hypoplastic nails on the fifth digit of the patient's fingers are shown.

vermilion of the lips, while patients with variant CSS collectively display a less coarse facial features, thinner eyebrows and thin vermillion border of the lips. However, both CSS and ARID1B-associated disorders have a phenotypic spectrum and cannot always be distinguished as two separate (classical or variant) categories. As a result of this phenotypic heterogeneity, no widely convincing clinical criteria and key diagnostic 
Table I. Primers and thermocycling conditions of quantitative-PCR for effective molecular concentration of the exome librarie.

\begin{tabular}{|c|c|c|c|c|}
\hline Primer & Sequence & Step 1 & Step 2 & Step 3 \\
\hline $\begin{array}{l}\text { Forward } \\
\text { Reverse }\end{array}$ & $\begin{array}{l}\text { 5'-AATGATACGGCGACCACCGA-3' } \\
\text { 5'-CAAGCAGAAGACGGCATACGA-3' }\end{array}$ & $\begin{array}{l}\text { Pre-denaturation } \\
\text { at } 95^{\circ} \mathrm{C} \text { for } 5 \mathrm{~min}\end{array}$ & $\begin{array}{l}\text { Denaturation at } 95^{\circ} \mathrm{C} \text { for } \\
30 \mathrm{sec} \text {, annealing at } 60^{\circ} \mathrm{C} \\
\text { for } 45 \mathrm{sec}, 35 \text { cycles }\end{array}$ & $\begin{array}{l}\text { Melt curve } \\
\text { analysis at } \\
65-95^{\circ} \mathrm{C}\end{array}$ \\
\hline
\end{tabular}

Table II. Primers and thermocycling conditions used for Sanger sequencing of the AT-rich interaction domain-containing protein $1 \mathrm{~B}$ gene.

\begin{tabular}{|c|c|c|c|c|}
\hline Primer & Sequence & Step 1 & Step 2 & Step 3 \\
\hline $\begin{array}{l}\text { Forward } \\
\text { Reverse }\end{array}$ & $\begin{array}{l}\text { 5'-GCCATCAGCAGGTTCCCTAA-3' } \\
\text { 5'-CGCCACTTACCAGGAGATGG-3' }\end{array}$ & $\begin{array}{l}\text { Predenaturation } \\
\text { at } 95^{\circ} \mathrm{C} \text { for } 5 \mathrm{~min}\end{array}$ & $\begin{array}{l}\text { Denaturation at } 95^{\circ} \mathrm{C} \text { for } \\
30 \mathrm{sec} \text {, annealing at } 60^{\circ} \mathrm{C} \\
\text { for } 30 \mathrm{sec} \text {, chain extension } \\
\text { at } 72^{\circ} \mathrm{C} \text { for } 30 \mathrm{sec}, 30 \text { cycles }\end{array}$ & $\begin{array}{l}\text { Final extension at } \\
72^{\circ} \mathrm{C} \text { for } 10 \mathrm{~min}\end{array}$ \\
\hline
\end{tabular}

II

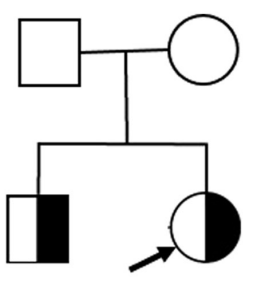

$(+/+)$

(c.3468_c.3471delCGAA/+)

Patient

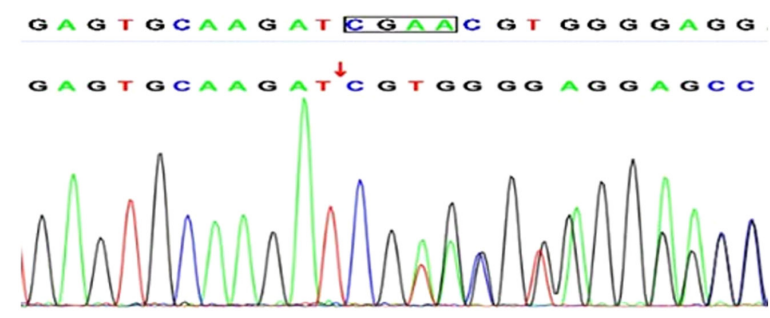

Father

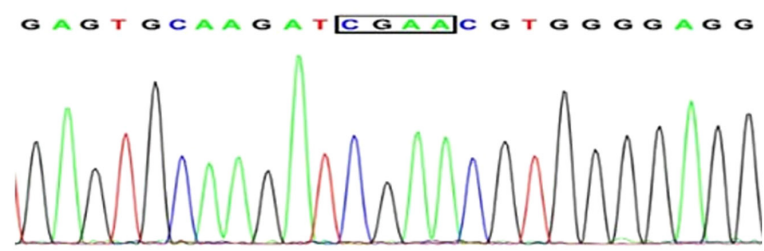

Mother

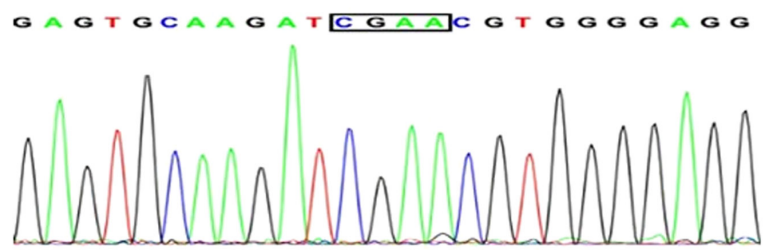

Older brother

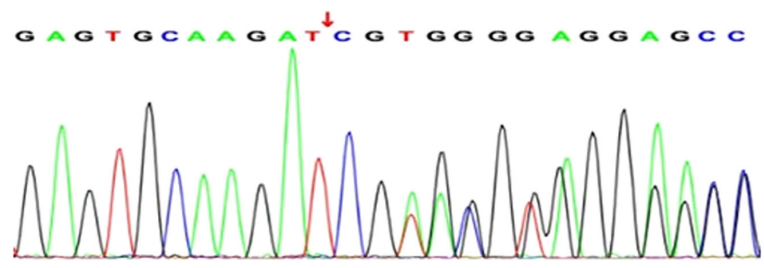

Figure 2. Pedigree of the family and DNA sequencing showed a heterozygous 4-bp deletion (c.3468_3471del) in the 13th exon of the ARID1B gene in the patient and her brother; this variant was not present in the parents. ARID1B, AT-rich interaction domain-containing protein 1B. 


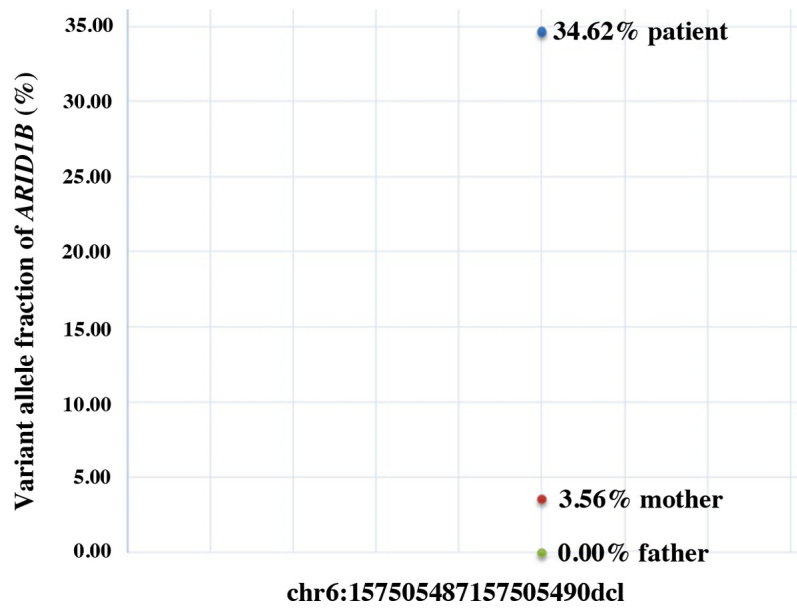

Figure 3. Amplicon-based deep sequencing demonstrated a 3.56\% somatic ARID1B mosaicism in the patient's mother. ARID1B, AT-rich interaction domain-containing protein $1 \mathrm{~B}$.

features have yet been outlined $(1,3,5,7,19,20)$. Thus, molecular testing currently has an important role in the diagnosis of CSS.

In 2012, CSS was reported to be caused by pathogenic variants in genes encoding subunits of the SWI/SNF complex, which function as chromatin remodeling factors (21-23). These include SMARCB1, SMARCA2, SMARCA4, SMARCE1, ARID1A and ARID1B at 22q11.23, 9p24.3, 19p13.2, 17q21.2, 1p36.11 and 6q25.3 (21-23). Additional genes (including SOX11, ADNP, PHF6 and TBC1D24 at 2p25.2, 20q13.13, Xq26.2 and 16p13.3, have alsobeen identified (24). Evidence has indicated that pathogenic variants in ARID1B are the primary genetic cause of CSS, accounting for $51-75 \%$ of cases $(3,5-7)$ in European, Japan, Canada, USA, between 2013-2019. However, ARID1B is the most frequently mutated gene in unspecified intellectual disability cohorts $(\sim 1 \%)$ from the United Kingdom and Germany in the past 8 years $(7,25,26)$. ARID1B pathogenic variants are associated with phenotypes that vary from non-syndromic intellectual disability to CSS (7).

The patient and her brother presented with intellectual disabilities, speech problems, developmental delay, hypoplastic fifth fingernails and/or toenails and brachytelephalangy. Additionally, the patient and her brother exhibited dysmorphic features, including coarse facial features, low hairline, bushy eyebrows, thick lips, scoliosis, body hypertrichosis and dental anomalies. The two siblings were clinically diagnosed with CSS1; this was confirmed using WES, which demonstrated heterozygous ARID1B variants [c.3468 (exon13)_c.3471 (exon13)_delCGAA] present in both siblings.

The majority of reported CSS1 cases are caused by heterozygous pathogenic variants in the ARID1B gene are sporadic $(5,8)$. However, exceptions have been noted, including a pathogenic variant of ARID1B that was passed down from an affected mother to her son (19). In a consanguineous Emirati family, a heterozygous variant of ARID1B was present in three affected siblings; however, this was absent in their parents and unaffected siblings (8). This indicated that one of the parents was a gonadal mosaic.

In the present study, the heterozygous pathogenic variant [c.3468 (exon13)_c.3471 (exon13) delCGAA] was identified using WES in two siblings; however, this was not initially observed in the parents. Therefore, it was hypothesized that this was a de novo pathogenic variant. Additionally, we hypothesized that one of the patient's parents exhibited gonadal mosaicism for the pathogenic variant, which has previously been reported (8); however, this had not been verified by laboratory tests. In order to test this hypothesis, amplicon-based deep sequencing was performed using samples from the patient's parents. An ARID1B pathogenic variant (4\%; chr6:157505487-157505490) was observed in the mother; however, this was absent in the father. This indicated that the mother exhibited the somatogonadal mosaic ARID1B variant. To the best of our knowledge, this is the first report based on experimental evidence of an ARID1B pathogenic variant in CSS1 that was inherited from the clinically healthy somatogonadal mosaic mother.

In summary, a heterozygous ARID1B pathogenic variant [c.3468 (exon13)_c.3471 (exon13)_delCGAA] was identified in the patient and her brother, both of whom exhibited the classical phenotype of CSS1. The results of the present study expand the spectrum of known ARID1B pathogenic variants, as well as the ethnic backgrounds of reported cases. To the best of our knowledge, the present study is the first to report two siblings with a pathogenic ARID1B variant inherited from their mother who exhibited somatogonadal mosaicism.

\section{Acknowledgements}

The authors would like to thank Miss Wenna Lin from Beijing Chigene Translational Medical Research Center Co. Ltd., Beijing, China. She provided the NGS test, genetic disease analyses and helpful discussion.

\section{Funding}

No funding was received.

\section{Availability of data and materials}

The datasets used and/or analyzed during the present study are available from the corresponding author on reasonable request.

\section{Authors' contributions}

ZM, CQ and DY conceived and designed the current study and analyzed data. ZM and DY prepared the manuscript. All authors read and approved the final manuscript.

\section{Ethics approval and consent to participate}

Ethical approval for the present study was obtained from the Institutional Review Board, Children's Hospital of Chongqing Medical University, Chongqing, China (approval no. 2018-64).

\section{Patient consent for publication}

Consent for publication of the patient's data/images in this paper was obtained from the parents.

\section{Competing interests}

The authors declare that they have no competing interests. 


\section{References}

1. Coffin GS and Siris E: Mental retardation with absent fifth fingernail and terminal phalanx. Am J Dis Child 119: 433-439, 1970.

2. Vergano SS and Deardorff MA: Clinical features, diagnostic criteria, and management of Coffin-Siris syndrome. Am J Med Genet C Semin Med Genet 166C: 252-256, 2014.

3. Wieczorek D, Bögershausen N, Beleggia F, Steiner-Haldenstätt S, Pohl E, Li Y, Milz E, Martin M, Thiele H, Altmüller J, et al: A comprehensive molecular study on Coffin-Siris and Nicolaides-Baraitser syndromes identifies a broad molecular and clinical spectrum converging on altered chromatin remodeling. Hum Mol Genet 22: 5121-5135, 2013.

4. Santen GW and Clayton-Smith J; ARID1B-CSS consortium: The ARID1B phenotype: What we have learned so far. Am J Med Genet C Semin Med Genet 166C: 276-289, 2014.

5. Santen GW, Aten E, Vulto-van Silfhout AT, Pottinger C, van Bon BW, van Minderhout IJ, Snowdowne R, van der Lans CA, Boogaard M, Linssen MM, et al: Coffin-Siris syndrome and the BAF complex: Genotype-phenotype study in 63 patients. Hum Mutat 34: 1519-1528, 2013.

6. Tsurusaki Y, Okamoto N, Ohashi H, Mizuno S, Matsumoto N, Makita Y, Fukuda M, Isidor B, Perrier J, Aggarwal S, et al: Coffin-Siris syndrome is a SWI/SNF complex disorder. Clin Genet 85: 548-554, 2014.

7. van der Sluijs PJ, Jansen S, Vergano SA, Adachi-Fukuda M, Alanay Y, AlKindy A, Baban A, Bayat A, Beck-Wödl S, Berry K, et al: The ARID1B spectrum in 143 patients: From nonsyndromic intellectual disability to Coffin-Siris syndrome. Genet Med 21: 1295-1307, 2019.

8. Ben-Salem S, Sobreira N, Akawi NA, Al-Shamsi AM, John A, Pramathan T, Valle D, Ali BR and Al-Gazali L: Gonadal mosaicism in ARID1B gene causes intellectual disability and dysmorphic features in three siblings. Am J Med Genet A 170A: 156-161, 2016.

9. Alfert A, Moreno N and Kerl K: The BAF complex in development and disease. Epigenetics Chromatin 12: 19, 2019.

10. Hargreaves DC and Crabtree GR: ATP-dependent chromatin remodeling: Genetics, genomics and mechanisms. Cell Res 21: 396-420, 2011.

11. Nie Z, Yan Z, Chen EH, Sechi S, Ling C, Zhou S, Xue Y, Yang D, Murray D, Kanakubo E, et al: Novel SWI/SNF chromatinremodeling complexes contain a mixed-lineage leukemia chromosomal translocation partner. Mol Cell Biol 23: 2942-2952, 2003.

12. Biesecker LG and Spinner NB: A genomic view of mosaicism and human disease. Nat Rev Genet 14: 307-320, 2013.

13. D'Gama AM and Walsh CA: Somatic mosaicism and neurodevelopmental disease. Nat Neurosci 21: 1504-1514, 2018.

14. Happle R: Gonosomal versus somatogonadal mosaicism: What is in a name. Am J Med Genet A 179: 1678, 2019.

15. Lim ET, Uddin M, De Rubeis S, Chan Y, Kamumbu AS, Zhang X, D'Gama AM, Kim SN, Hill RS, Goldberg AP, et al: Rates, distribution and implications of postzygotic mosaic mutations in autism spectrum disorder. Nat Neurosci 20: 1217-1224, 2017.
16. Wright CF, Prigmore E, Rajan D, Handsaker J, McRae J Kaplanis J, Fitzgerald TW, FitzPatrick DR, Firth HV and Hurles ME: Clinically-relevant postzygotic mosaicism in parents and children with developmental disorders in trio exome sequencing data. Nat Commun 10: 2985, 2019.

17. Watkins MW and Beaujean AA: Bifactor structure of the wechsler preschool and primary scale of intelligence-fourth edition. Sch Psychol Q 29: 52-63, 2014.

18. Richards S, Aziz N, Bale S, Bick D, Das S, Gastier-Foster J, Grody WW, Hegde M, Lyon E, Spector E, et al: Standards and guidelines for the interpretation of sequence variants: A joint consensus recommendation of the American college of medical genetics and genomics and the association for molecular pathology. Genet Med 17: 405-424, 2015.

19. Mannino EA, Miyawaki H, Santen G and Schrier Vergano SA: First data from a parent-reported registry of 81 individuals with Coffin-Siris syndrome: Natural history and management recommendations. Am J Med Genet A 176: 2250-2258, 2018

20. Vergano SA, van der Sluijs PJ and Santen G: ARID1B-Related Disorder (ARID1B-RD). GeneReviews ${ }^{\circledR}$ [Internet], University of Washington, Seattle, WA, 1993-2021.

21. Santen GW, Aten E, Sun Y, Almomani R, Gilissen C, Nielsen M, Kant SG, Snoeck IN, Peeters EA, Hilhorst-Hofstee Y, et al: Mutations in SWI/SNF chromatin remodeling complex gene ARID1B cause Coffin-Siris syndrome. Nat Genet 44: 379-380, 2012.

22. Smith JA, Holden KR, Friez MJ, Jones JR and Lyons MJ: A novel familial autosomal dominant mutation in ARID1B causing neurodevelopmental delays, short stature, and dysmorphic features. Am J Med Genet A 170: 3313-3318, 2016.

23. Tsurusaki Y, Okamoto N, Ohashi H, Kosho T, Imai Y, Hibi-Ko Y, Kaname T, Naritomi K, Kawame H, Wakui K, et al: Mutations affecting components of the SWI/SNF complex cause Coffin-Siris syndrome. Nat Genet 44: 376-378, 2012.

24. Kosho T, Miyake N and Carey JC: Coffin-Siris syndrome and related disorders involving components of the BAF (mSWI/SNF) complex: Historical review and recent advances using next generation sequencing. Am J Med Genet C Semin Med Genet 166C: 241-251, 2014.

25. Wright CF, Fitzgerald TW, Jones WD, Clayton S, McRae JF, van Kogelenberg M, King DA, Ambridge K, Barrett DM, Bayzetinova T, et al: Genetic diagnosis of developmental disorders in the DDD study: A scalable analysis of genome-wide research data. Lancet 385: 1305-1314, 2015.

26. Hoyer J, Ekici AB, Endele S, Popp B, Zweier C, Wiesener A, WohlleberE,Dufke A, RossierE,Petsch C, et al:Haploinsufficiency of ARID1B, a member of the SWI/SNF-a chromatin-remodeling complex, is a frequent cause of intellectual disability. Am J Hum Genet 90: 565-572, 2012.

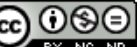

This work is licensed under a Creative Commons Attribution-NonCommercial-NoDerivatives 4.0 International (CC BY-NC-ND 4.0) License. 